Lee, J. \& Strazicich, M. (2001). Testing the Null of Stationarity in the Presence of a Structural Break. Applied Economics Letters, 8(6): 377-382 (June 2001). Published by Taylor \& Francis (ISSN: 1350-4851). DOI: $10.1080 / 135048501750237810$.

\title{
Testing the null of stationarity in the presence of a structural break
}

Junsoo Lee and Mark C. Strazicich

\author{
ABSTRACT \\ A test for stationarity in the presence of a structural break is proposed. An unknown \\ break point is endogenously determined at the value minimizing the test statistic. \\ The break point can be estimated reasonably well under the null hypothesis of \\ stationarity, especially when the magnitude of the break is large.
}




\section{I . INTRODUCTION}

The pioneering work of Perron (1989) illustrates the need to allow for a structural break when testing for a unit root in economic time series. Since Perron's break point is given exogenously, subsequent literature has incorporated an endogenous break point. Allowing for a break point endogenously determined from the data, Zivot and Andrews (1992), and others, consider a minimum unit root statistic for which a break point is determined at the level giving the minimum $t$-statistic. These tests examine the null hypothesis of a unit root in the presence of a structural break. Lee et al. (1997) have shown that tests examining the null of stationarity are also affected by the presence of a structural break. They show that the stationarity tests of

Kwiatkowski et al. (1992, hereafter denoted KPSS) diverge in the presence of an unaccounted for structural break. This finding illustrates the need for developing stationary tests that allow for a structural break.

This paper proposes a version of the minimum test for the null hypothesis of stationarity in the presence of a structural break. To determine an unknown break point endogenously from the data, a minimum statistic is considered for which the break point is selected at the value minimizing the test statistic. The performance of the test is then investigated, and as to how well it can detect an unknown break point is examined. This task is more meaningful when testing for stationarity than when testing for a unit root, since the null hypothesis of the former implies a stationary process involving a break, while the null distribution of the latter is shown not to depend on the break point. Throughout the paper, ' $\rightarrow$ ' indicates weak convergence as $T \rightarrow \infty$. 


\section{I . TEST STATISTICS WITH A FIXED BREAK POINT}

As in KPSS, an unobserved components representation is considered in which a time series $y_{t}, t=1,2, \ldots, T$, is decomposed into the sum of a random walk and a stationary error term as follows:

$$
\begin{aligned}
& y_{t}=r_{t}+\varepsilon_{t} \\
& r_{t}=r_{t-1}+u_{t}
\end{aligned}
$$

where $\varepsilon_{t}$ is assumed stationary and $u_{t}$ is $i d d\left(0, \sigma_{u}^{2}\right)$. KPSS test the null hypothesis of stationarity around a level $r_{0}$ by examining the null hypothesis $\sigma_{u}^{2}=0$. When $r_{t}=\gamma+r_{r_{t-1}+u_{t}}$ the null hypothesis implies stationarity around the trend function $\gamma t$.

To allow for a structural break, the following models using different specifications for $r_{t}$ are considered.

$$
\begin{aligned}
& \left(\mathrm{M}_{1}\right)^{*} r_{t}=\delta_{1} B_{t}+r_{t-1}+u_{t} \\
& \left(\mathrm{M}_{2}\right)^{*} r_{t}=\gamma+\delta_{1} B_{t}+\delta_{2} D_{t}+r_{t-1}+u_{t}
\end{aligned}
$$

where $B_{t}=1$ for $t=T_{B}+1$ and zero otherwise; $D_{t}=1$ for $t \geqslant T_{B}+1$ and zero otherwise; and $T_{B}$ stands for the time period when a structural change occurs. The following corresponding models are then considered.

$$
\begin{aligned}
& \left(\mathrm{M}_{1}\right) y_{t}=\alpha+\delta_{1} D_{t}+e_{t} \\
& \left(\mathrm{M}_{2}\right) y_{t}=\alpha+\gamma t+\delta_{1} D_{t}+\delta_{2} D T_{t}^{*}+e_{t}
\end{aligned}
$$

where $D T_{t}^{*}=t-T_{B}$ for $t \geqslant T_{B}+1$ and zero otherwise. Perron (1989) previously considered these models. $\mathbf{M}_{1}$ describes a stationary process with a one-time shift in the level and $\mathrm{M}_{2}$ allows for a sudden change in the level followed by a change in the slope of the trend function.

Here stationarity tests that allow for a structural break are proposed. For the time being, it is assumed as in Perron (1989), that the break point is fixed and known a priori. Consequently, the DGP and the test regressions are referred to as either $M_{1}$ in (3a) for a level stationarity test, or as $\mathrm{M}_{2}$ in (3b) for a trend stationarity test. The null hypothesis implies stationarity around a break point for $\mathrm{M}_{1}$, or stationarity around a trend function for $\mathrm{M}_{2}$. The level stationarity test statistic $\tilde{\eta}_{\mu}$ allowing for an exogenous break is given by:

$$
\tilde{\eta}_{\mu}=T^{-2} \sum_{t=1}^{T} \hat{S}_{t}^{2} / s^{2}(l)
$$


The residuals $\tilde{\boldsymbol{e}}_{t}$ from the regression (3a) are used to construct $\tilde{S}_{t}=\sum_{j=1}^{t} \tilde{e}_{j} . \tilde{S}^{2}(l)$ is the estimate of the long-run variance $\sigma^{2}=\lim T^{-1}\left(\sum e_{t}\right)^{2}$. The long-run variance is constructed via non-parametric estimation of $\hat{s}^{2}(l)=\hat{\Gamma}_{0}+2 \sum w_{j} \hat{\Gamma}_{j}$ by choosing a truncation lag parameter $l$ and a set of weights $w_{j}, j=1, \ldots, l$, where $\Gamma_{j}$ is the $j$ th sample autocovariance of the residuals $\tilde{e}_{t}$. The corresponding trend stationarity test statistic $\tilde{\eta}_{\tau}$ is obtained in the same manner from the regression (3b).

The next theorem states the asymptotic null distribution of the $\tilde{\eta}_{\mu}$ statistic under the strong mixing regularity conditions of Phillips and Perron (1988, p. 336).

Theorem 1. Suppose that the DGP is subject to a structural break as in (3a). Under the null hypothesis of stationarity around the break, $\sigma_{u}^{2}=0$,

$$
\tilde{\eta}_{\mu} \rightarrow \lambda^{2} \int_{0}^{1} V_{1}\left(b_{1}\right)^{2} d b_{1}+(1-\lambda)^{2} \int_{0}^{1} V_{2}\left(b_{2}\right)^{2} d b_{2}
$$

where $V_{1}$ and $V_{2}$ are two independent Brownian bridges; $V_{i}\left(b_{i}\right)=W\left(b_{i}\right)-b_{i} W(1)$, for $i=1,2$, with $0<b_{1}=$ $b / \lambda<1$ and $0<b_{2}=(b-\lambda) /(1-\lambda)<0$, where $W$ is a Brownian motion.

Proof. See Lee (1996b).

For the $\tilde{\eta}_{\tau}$ statistic, a partial sum of the residuals is obtained from the regression (3b). The resulting asymptotic distribution is the same as in expression (5), except that the term $V_{i}\left(b_{i}\right)$ is replaced by the second-level Brownian bridge whose expression appears in KPSS (1992, p. 167). The limiting distribution is expressed as a weighted sum of two independent terms. Each of them can be obtained from the regression using the subsample of before or after the structural break. The symmetry of the distribution around $\lambda=0.5$ is easily observed, since we can interchange $\lambda$ and $(1-\lambda)$ in this case. The asymptotic distributions of the test statistics $\tilde{\eta}_{\mu}$ and $\tilde{\eta}_{\tau}$ can be simulated accordingly. Critical values are calculated via Monte Carlo simulation using a sample size of 2000 with 50000 replications. Results are shown in Table 1. 
Table 1. Upper tail critical values for $\bar{\eta}_{\mu}$ and $\bar{\eta}_{T}$

\begin{tabular}{lcccc}
\hline$\lambda$ & $10 \%$ & $5 \%$ & $2.5 \%$ & $1 \%$ \\
\hline \multicolumn{4}{l}{ Upper tail percentiles of the distribution of $\bar{\eta}_{\mu}$} \\
0.1 & 0.2880 & 0.3809 & 0.4712 & 0.6009 \\
0.2 & 0.2301 & 0.3028 & 0.3819 & 0.4842 \\
0.3 & 0.1880 & 0.2452 & 0.3064 & 0.3878 \\
0.4 & 0.1597 & 0.2004 & 0.2431 & 0.3003 \\
0.5 & 0.1531 & 0.1891 & 0.2256 & 0.2690 \\
\multicolumn{5}{l}{ Upper tail percentiles of the distribution of $\bar{\eta}_{\tau}$} \\
0.1 & 0.0981 & 0.1223 & 0.1467 & \\
0.2 & 0.0794 & 0.0984 & 0.1171 & 0.1788 \\
0.3 & 0.0654 & 0.0792 & 0.0933 & 0.1429 \\
0.4 & 0.0556 & 0.0656 & 0.0761 & 0.1134 \\
0.5 & 0.0528 & 0.0615 & 0.0701 & 0.0904 \\
\hline
\end{tabular}

\section{I I. TEST STATISTICS WITH AN UNKNOWN BREAK POINT}

In practical estimation, one rarely knows the break point $T_{B}$ a priori. To determine an unknown break point endogenously from the data, a minimum stationarity test is considered. The estimation scheme for the minimum stationarity test is to choose a break point that gives the most favorable result for the null of stationarity around a break. This means that the estimate of $\lambda$ is obtained at the value that minimizes the stationarity statistic. This scheme diOEers in principle from that of the minimum unit root test that adopts the least favourable result for the null hypothesis. Both the minimum stationarity test and the minimum unit root test share the notion of taking the minimum of the statistics over a range of $\lambda$ between 0 and 1 . It appears obvious that a supreme test is not appropriate for testing stationarity, because it tends to maximize the error sum of squares. Therefore, we consider minimum level and trend stationarity statistics with an endogenous break as follows:

$$
\begin{aligned}
& \operatorname{Inf} \tilde{\eta}_{\mu}=\inf _{\lambda \in \Lambda} \tilde{\eta}_{\mu}(\lambda) \\
& \operatorname{Inf} \tilde{\eta}_{\tau}=\inf _{\lambda \in \Lambda} \tilde{\eta}_{\tau}(\lambda)
\end{aligned}
$$


where $\Lambda$ is a closed subset of $(0,1)$ and $\tilde{\lambda}=\tilde{T}_{B} / T . \tilde{T}_{B}$ is the estimated break point from a sample of size $T$. Here, $\tilde{\lambda}$ has a well-defined probability distribution over $\Lambda$, and the true value of $\lambda$ is included in the sample space of $\tilde{\lambda}$. The same is true for $\tilde{T}_{B}$ defined over $(0,1, \ldots, T)$. Then the asymptotic distribution of the minimum statistic $\operatorname{Inf} \tilde{\eta}_{\mu}$ is given by:

$$
\int_{0}^{1} \Phi\left[\tilde{\eta}_{\mu}(\tilde{\lambda}) \mid \tilde{\lambda}=\lambda\right] P(\tilde{\lambda}=\lambda) d \lambda
$$

where $\Phi\left[\tilde{\eta}_{\mu}(\tilde{\lambda}) \mid \tilde{\lambda}=\lambda\right]$ is the asymptotic distribution of Inf $\tilde{\eta}_{\mu}$ conditional on $\tilde{\lambda}=\lambda$, and $P(\tilde{\lambda}=\lambda)$ is the probability that $\tilde{\lambda}=\lambda$. The asymptotic distribution of $\operatorname{Inf} \tilde{\eta}_{\tau}$ is obtained in a similar manner.

As an extreme case, suppose that $P(\tilde{\lambda}=\lambda)=1$ and a break point is correctly estimated. Then the null distribution of $\operatorname{Inf} \tilde{\eta}_{\mu}$ approaches asymptotically that of $\tilde{\eta}_{\mu}$, which assumes an exogenous break.

Corollary 1. Suppose that $\tilde{T}_{B} \rightarrow T_{B}$ and $\tilde{\lambda} \rightarrow \lambda$ as $T \rightarrow \infty$. Then, the asymptotic null distribution of the Inf $\tilde{\eta}_{\mu}$ statistic converges to that of the $\tilde{\eta}_{\mu}$ statistic.

Proof. See Lee (1996b).

The same is true for the $\operatorname{Inf} \tilde{\eta}_{\tau}$ test. It is worth noticing that the asymptotic null distribution of $\operatorname{Inf} \tilde{\eta}_{\mu}$ is not free of $\lambda$. An intuitive reason is that the null hypothesis of the stationarity statistic should imply $\lambda$. This property differs from that of the minimum unit root test of Zivot and Andrews (1992), since the asymptotic distribution of the minimum unit root statistic is free of $\lambda .{ }^{1}$ The method of the minimum stationarity test can be accordingly viewed as a procedure for identifying a break point under the null of stationarity. The break point needs to be correctly estimated to have correct size under the null of stationarity. On the other hand, the minimum unit root test allows us to identify a break point when the alternative hypothesis is stationary. In their test, a break point must be correctly estimated to increase power.

When testing for stationarity, a break point cannot be consistently estimated under the alternative hypothesis of a unit root. Nunes et al. (1995) provide evidence of the difficulty of obtaining a precise ML estimate of a break point when an integrated process is involved. The same is true for the minimum stationarity test under the alternative unit root hypothesis. However, the difficulty of estimating $\lambda$ 
does not negate the validity of the minimum stationarity test, since the test statistic simply diverges under the alternative. Thus, stationarity tests have power to reject the null of stationarity if the DGP implies the alternative hypothesis.

Simply put, we can say that it is only the estimated break point that is not reliable when the stationary null hypothesis is rejected.

\section{PERFORMANCE}

This section shows Monte Carlo simulation results on the performance of the stationarity statistic. Because the behaviour of the trend stationarity tests $\tilde{\eta}_{\tau}$ and $\operatorname{Inf} \tilde{\eta}_{\tau}$ is not much different from those of the level stationarity tests $\tilde{\eta}_{\mu}$ and $\operatorname{Inf} \tilde{\eta}_{\mu}$, we focus on the latter in the simulations. Pseudo-iid $\mathrm{N}(0,1)$ random numbers were generated using Gauss procedure RNDNS, and all calculations were conducted using the Gauss software version 3.1.4. The DGP implies (3a). Initial values $y_{0}$ and $e_{0}$ are taken a random numbers, and assume that $e_{t}$ are i.i.d. and $\sigma_{e}^{2}=1$. The Gauss application software COINT version 2.0 (Ouliaris and Phillips, 1994) is used to obtain the estimate of the long-run variance in Equation 4. For the choice of a truncation lag, we employ three fixed values of $l: l_{0}=0$, $l_{4}=\operatorname{int}\left[4(T / 100)^{1 / 4}\right]$, and $l_{12}=\operatorname{int}\left[12 /(T / 100)^{1 / 4}\right]$, where 'int' takes the value of the nearest integer to the expression in parentheses. The optimal bandwidth selection procedure of Andrews (1991) is also employed. The pre-whitening procedure of Andrews and Monahan (1992) is not used, since it makes stationarity tests inconsistent. ${ }^{2}$ The choice of lag window does not significantly change the results and the Fejer lag window will be used here. All simulation results are calculated using 5000 replications and the size (rejection frequencies at the 5\% level when the null hypothesis is true) and power (rejection frequencies when the alternative hypothesis is true) of the tests are evaluated using the upper $5 \%$ critical value. 


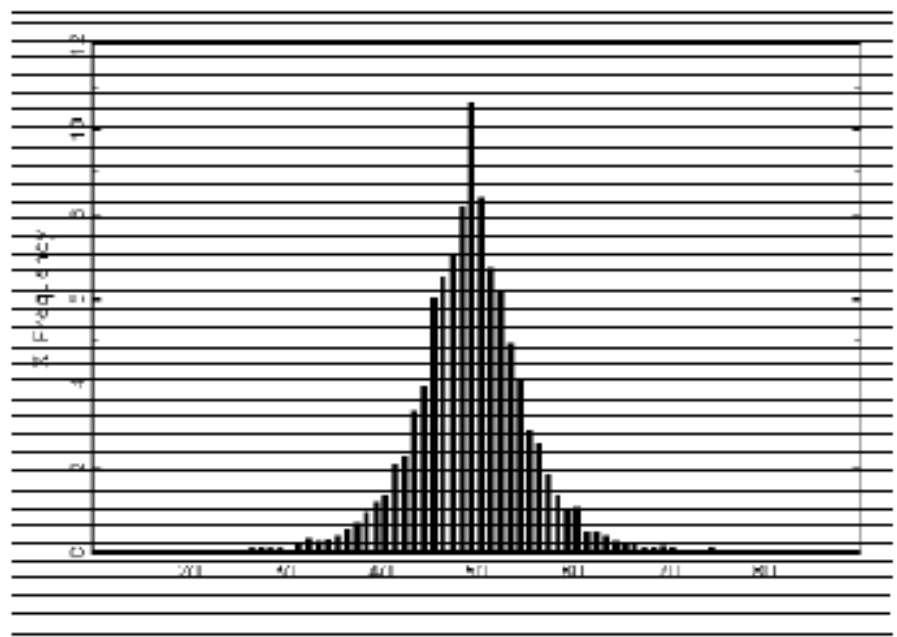

(a) $d=1$

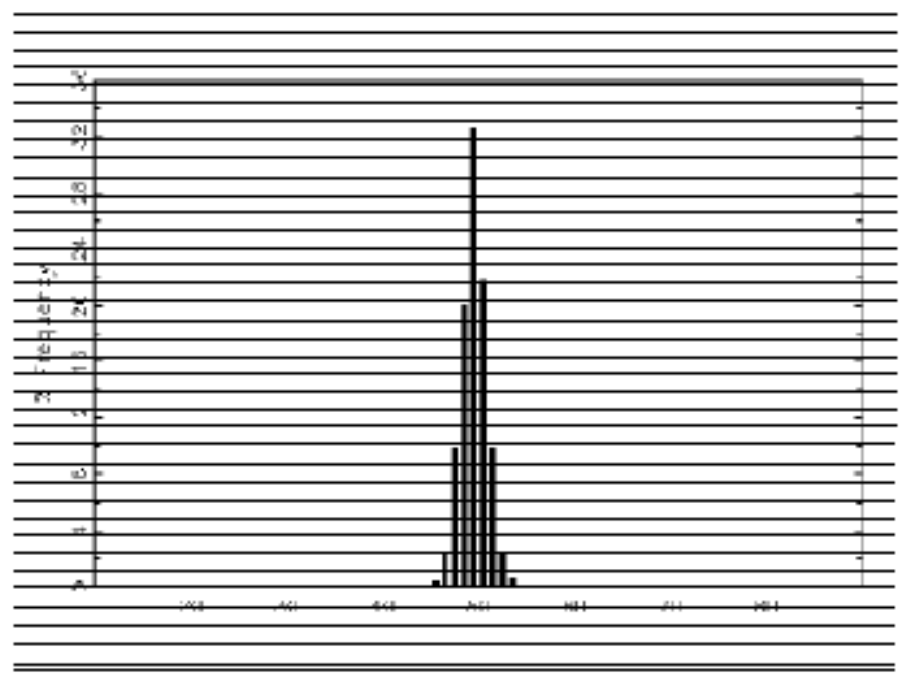

(b) $d=3$

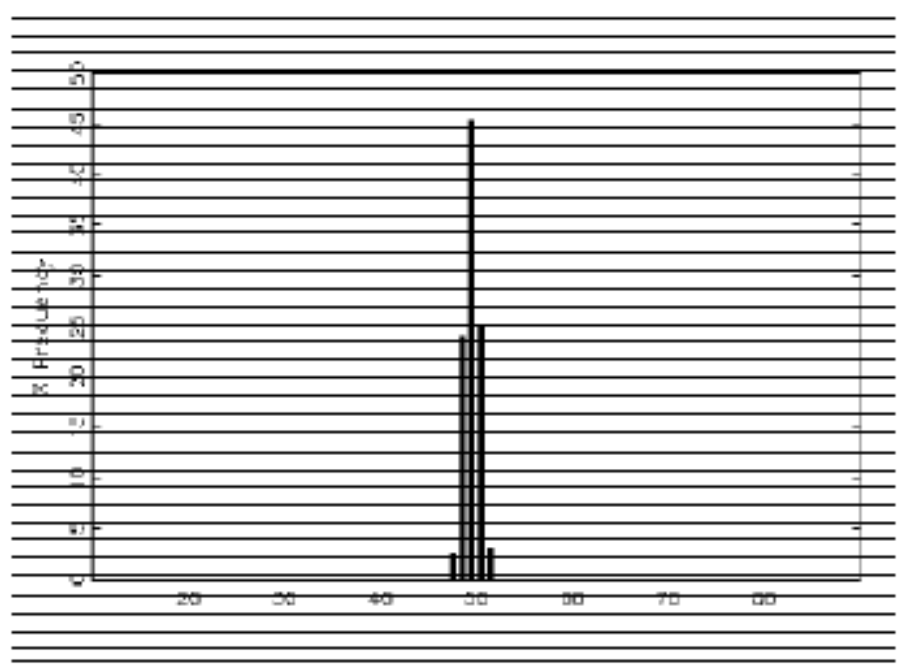

(c) $d=5$

Fig. 1. Empirical distribution of estimated break points 
The performance of the statistics under the null hypothesis of stationarity is examined. The sample size is given as $T=100$. Results are reported in Table 2. Experiment A examines whether $\tilde{\eta}_{\mu}$ and $\operatorname{Inf} \tilde{\eta}_{\mu}$ are invariant to changes of a structural break under iid errors for which using $l_{0}$ is most appropriate. Results from both tests show that size is not affected by the location of the break. The size remains similar when $\lambda=0.5$ or 0.2 . In addition, $\tilde{\eta}_{\mu}$ is mostly invariant to the different magnitudes of the level shift parameter $d$, where $d=1,2$, or 3 . This is an important result, indicating that the stationarity test statistic $\tilde{\eta}_{\mu}$ does not depend on the parameters describing the break. As expected, Inf $\tilde{\eta}_{\mu}$ is affected by the magnitude of $d$. The Inf $\tilde{\eta}_{\mu}$ test rejects the true null hypothesis less often than it should when $d$ is small. This result occurs because $P(\tilde{\lambda}=\lambda)$ departs from one. This phenomenon, though not critical, leads to a mild size distortion. However, as $d$ gets bigger the size of the test gets closer to its nominal size

of $5 \%$. Figure 1 provides empirical distributions of the estimated break point $\tilde{\lambda}$ for different values of $d$. The empirical distribution shows that $\tilde{\lambda}$ centers on the true value of $\lambda(0.5)$ in the DGP, while its variance gets smaller as $d$ increases. When $d$ is large enough the estimated $\tilde{\lambda}$ converges to a mass point $\lambda$, and the distribution of the corresponding Inf $\tilde{\eta}_{\mu}$ test would be the same as that of the $\tilde{\eta}_{\mu}$ test, which assumes a given fixed break point. This result indicates that the distribution of $\operatorname{Inf} \tilde{\eta}_{\mu}$ approaches asymptotically that of $\tilde{\eta}_{\mu}$ as $d$ gets larger. In the finite sample, however, the distribution is somewhat dependent on the degree of accuracy of estimating the true break. In this sense, though not critical it is recommended in the finite sample to use adjusted bootstrapping critical values to avoid size distortion, when the estimated break coefficient is relatively small.

Experiment $\mathrm{B}$ investigates the size of the tests in the presence of autocorrelated errors. We consider AR(1) errors of the form $e_{t}=\rho e_{t-1}+v_{t}$, where $v_{t}$ is i.i.d. Table 2 presents the results for $\rho=0, \pm 0.2,+0.5$, and \pm 0.8 , using various lag selections. Results with $l_{0}$ and $l_{4}$ lags exhibit non-negligible size distortions for $\rho>0.5$, but the results using $l_{12}$ and the data driven optimal bandwidth lags are fairly good. Results are comparable to the KPSS test in the absence of a break (KPSS, Table 3). As in KPSS, even the tests using optimal lags exhibit noticeable distortions when errors are strongly and negatively correlated, especially for $\rho=-0.8$. 
Experiment $\mathrm{C}$ considers the power of the tests. We report simulation results in Table 3 for different values of $\sigma_{u}^{2}$ (with

$\sigma_{\varepsilon}^{2}=1$ ) under the assumption of iid errors, so that the power comparison would not be affected by potential size distortions. As explained in KPSS, the power of the test increases as $T$ increases. ${ }^{3}$ The power of the tests using the optimal bandwidth lags is somewhat lower than the power of the tests using $l_{0}$ and $l_{4}$ lags. In some cases, it is lower than that of the tests using $l_{12}$ lags. It is expected that using $l_{0}$ or $l_{4}$ lags produces better power, since the optimal choice of the truncation lag under iid errors is zero $\left(l_{0}\right)$, and estimating with too many lags typically results in a loss of power.

\section{SUMMARY}

In this paper a minimum stationarity test in the presence of a structural break has been proposed. An unknown break is endogenously determined at the point minimizing the stationarity test statistic. It is shown that the minimum stationarity test performs reasonably well in identifying the unknown break point especially when the magnitude of the structural break is large. Under the alternative hypothesis of a unit root, the minimum stationarity test cannot detect the break point precisely, but this difficulty does not negate the validity of the test. The test is shown to have sufficient power to reject the null hypothesis of stationarity, whether or not the break point is precisely estimated. 
Table 2. Size of stationarity tests

\begin{tabular}{|c|c|c|c|c|c|c|c|c|c|c|c|}
\hline \multirow[b]{2}{*}{ Exp } & \multirow[b]{2}{*}{$\lambda$} & \multirow[b]{2}{*}{$d$} & \multirow[b]{2}{*}{$\rho$} & \multicolumn{4}{|l|}{$\bar{\eta}_{\mu}$} & \multicolumn{4}{|l|}{$\operatorname{Inf} \bar{\eta}_{\mu}$} \\
\hline & & & & $l_{0}$ & $l_{4}$ & $l_{12}$ & opt & $l_{0}$ & $l_{4}$ & $l_{12}$ & opt \\
\hline \multirow[t]{6}{*}{ A } & 0.5 & 1 & 0 & 0.050 & 0.039 & 0.031 & 0.046 & 0.005 & 0.002 & 0.001 & 0.003 \\
\hline & & 3 & & 0.051 & 0.039 & 0.032 & 0.046 & 0.011 & 0.014 & 0.013 & 0.011 \\
\hline & & 5 & & 0.053 & 0.039 & 0.031 & 0.049 & 0.014 & 0.006 & 0.014 & 0.015 \\
\hline & 0.2 & 1 & 0 & 0.052 & 0.053 & 0.053 & 0.047 & 0.006 & 0.000 & 0.000 & 0.003 \\
\hline & & 3 & & 0.052 & 0.051 & 0.054 & 0.052 & 0.012 & 0.000 & 0.000 & 0.001 \\
\hline & & 5 & & 0.047 & 0.059 & 0.046 & 0.057 & 0.009 & 0.000 & 0.000 & 0.001 \\
\hline \multirow[t]{14}{*}{ B } & 0.5 & 1 & 0 & 0.050 & 0.039 & 0.031 & 0.046 & 0.006 & 0.003 & 0.000 & 0.002 \\
\hline & & & 0.8 & 0.931 & 0.318 & 0.054 & 0.060 & 0.593 & 0.010 & 0.001 & 0.000 \\
\hline & & & 0.5 & 0.509 & 0.103 & 0.037 & 0.079 & 0.138 & 0.103 & 0.000 & 0.002 \\
\hline & & & 0.2 & 0.153 & 0.051 & 0.032 & 0.074 & 0.024 & 0.003 & 0.000 & 0.004 \\
\hline & & & -0.2 & 0.009 & 0.029 & 0.030 & 0.028 & 0.000 & 0.000 & 0.001 & 0.001 \\
\hline & & & -0.5 & 0.000 & 0.015 & 0.028 & 0.019 & 0.000 & 0.003 & 0.001 & 0.000 \\
\hline & & & -0.8 & 0.000 & 0.002 & 0.022 & 0.045 & 0.000 & 0.000 & 0.000 & 0.000 \\
\hline & 0.5 & 3 & 0 & 0.051 & 0.039 & 0.032 & 0.046 & 0.011 & 0.014 & 0.000 & 0.011 \\
\hline & & & 0.8 & 0.928 & 0.331 & 0.054 & 0.057 & 0.778 & 0.037 & 0.005 & 0.008 \\
\hline & & & 0.5 & 0.520 & 0.095 & 0.036 & 0.078 & 0.245 & 0.019 & 0.004 & 0.000 \\
\hline & & & 0.2 & 0.162 & 0.056 & 0.034 & 0.073 & 0.062 & 0.007 & 0.004 & 0.017 \\
\hline & & & -0.2 & 0.011 & 0.029 & 0.033 & 0.029 & 0.001 & 0.005 & 0.010 & 0.005 \\
\hline & & & -0.5 & 0.000 & 0.017 & 0.029 & 0.017 & 0.000 & 0.004 & 0.009 & 0.002 \\
\hline & & & -0.8 & 0.000 & 0.002 & 0.021 & 0.042 & 0.000 & 0.000 & 0.002 & 0.002 \\
\hline
\end{tabular}

Notes. 'opt' refers to the case where the optimal bandwidth procedure is used

$\left(\sigma_{u}^{2}=0, \operatorname{AR}(1)\right.$ error; $\left.T=100\right)$.

Table 3. Power of stationarity tests

\begin{tabular}{|c|c|c|c|c|c|c|c|c|c|c|c|}
\hline \multirow[b]{2}{*}{ Exp } & \multirow[b]{2}{*}{$\lambda$} & \multirow[b]{2}{*}{$d$} & \multirow[b]{2}{*}{$\sigma_{u}^{2}$} & \multicolumn{4}{|l|}{$\bar{\eta}_{\mu}$} & \multicolumn{4}{|l|}{$\operatorname{Inf} \bar{\eta}_{\mu}$} \\
\hline & & & & $l_{0}$ & $l_{4}$ & $l_{12}$ & opt & $l_{0}$ & $l_{4}$ & $l_{12}$ & opt \\
\hline \multirow[t]{10}{*}{ C } & 0.5 & 1 & 0.001 & 0.048 & 0.040 & 0.033 & 0.044 & 0.003 & 0.000 & 0.000 & 0.002 \\
\hline & & & 0.1 & 0.428 & 0.339 & 0.176 & 0.362 & 0.121 & 0.059 & 0.032 & 0.107 \\
\hline & & & 1 & 0.995 & 0.859 & 0.512 & 0.388 & 0.923 & 0.377 & 0.151 & 0.153 \\
\hline & & & 100 & 0.999 & 0.871 & 0.533 & 0.356 & 0.972 & 0.437 & 0.174 & 0.149 \\
\hline & & & 10000 & 0.999 & 0.867 & 0.526 & 0.355 & 0.973 & 0.487 & 0.151 & 0.128 \\
\hline & 0.5 & 3 & 0.001 & 0.045 & 0.042 & 0.037 & 0.045 & 0.003 & 0.000 & 0.000 & 0.002 \\
\hline & & & 0.1 & 0.424 & 0.341 & 0.184 & 0.396 & 0.192 & 0.079 & 0.050 & 0.114 \\
\hline & & & 1 & 0.994 & 0.860 & 0.525 & 0.386 & 0.942 & 0.375 & 0.177 & 0.183 \\
\hline & & & 100 & 0.999 & 0.871 & 0.530 & 0.373 & 0.973 & 0.388 & 0.152 & 0.130 \\
\hline & & & 10000 & 0.999 & 0.867 & 0.532 & 0.365 & 0.975 & 0.411 & 0.162 & 0.147 \\
\hline
\end{tabular}

Notes. $\sigma_{u}^{2}>0 ;$ did errors; $T=100$

\section{NOTES}

1 Recently, the assumption of no structural break under the null of a unit root for the Zivot and Andrews test has been criticized by Lee et al. (1998). This assumption might be necessary to have the null distribution free of $\lambda$ Since the null hypothesis does not include a break, rejection of the null hypothesis does not imply rejection of a unit root, but implies rejection of a unit root without break, potentially leading to spurious rejections of the unit root null.

2 See Lee (1996a) for further discussion.

3 Results using other sample sizes are not reported here, but are available upon request. 


\section{REFERENCES}

Andrews, D. W. K. (1991) Heteroskedasticity and autocorrelation consistent covariance matrix estimation, Econometrica, 59, $817 \pm 58$.

Andrews, D. W. K. and Monahan, J. C. (1992) An improved heteroskedasticity and autocorrelation consistent covariance matrix estimation, Econometrica, 60, 953 \pm 66 .

Kwiatkowski, D., Phillips, P. C. B., Schmidt, P. and Shin, Y. (1992) Testing the null hypothesis of stationarity against the alternative of a unit root: how sure are we that economic time series have a unit root? Journal of Econometrics, 54, $159 \pm 78$.

Lee, J., List, J. and Strazicich, M. (1998) Spurious rejections with the minimum unit root test in the presence of a structural break under the null, working paper, Department of Economics, University of Central Florida.

Lee, J. (1996a) On the power of stationarity tests using optimal bandwidth estimates, Economics Letters, 51, 131 7.

Lee, J. (1996b) Minimum statistics testing for stationarity in the presence of a structural break, working paper, Department of Economics, Vanderbilt University.

Lee, J., Huang, C. and Shin, Y. (1997) On stationarity tests in the presence of structural breaks, Economics Letters, 55, $165 \pm 72$. Nunes, L., Kuan, C. and Newbold, P. (1995) Spurious break, Econometric Theory, 11, 736 49 .

Ouliaris, S. and Phillips, P. C. B. (1994) Coint 2.0, Gauss

procedures for cointegrated regressions. Predicta Software Inc.

Perron, Pierre (1989) The great crash, the oil price shock, and the unit root hypothesis, Econometrica, 57, 1361 401.

Phillips, P. C. B. and Perron, P. (1988) Testing for a unit root in time series regression, Biometrika, 75, 335 46.

Zivot, Eric and Andrews, D. W. K. (1992) Further evidence on the great crash, the oil-price shock, and the unit-root hypothesis, Journal of Business and Economic Statistics, 10, 251 70. 